Article

\title{
Nanostructured Tungsten Oxide Composite for High-Performance Gas Sensors
}

\author{
Siyuan Feng-Chen ${ }^{1}$, Ali Aldalbahi ${ }^{2}$ and Peter Xianping Feng ${ }^{3, *}$ \\ 1 Escuela Secundaria de la Universidad de Puerto Rico, San Juan, PR 00936, USA; \\ E-Mail: sfengpr@gmail.com \\ 2 Department of Chemistry, King Saud University, Riyadh 11451, Saudi Arabia; \\ E-Mail: aaldalbahi@ksu.edu.sa \\ 3 Department of Physics, University of Puerto Rico, San Juan, PR 00936, USA \\ * Author to whom correspondence should be addressed; E-Mail: peter.feng@upr.edu; \\ Tel.: +1-787-764-2626; Fax: +1-787-764-4063.
}

Academic Editor: Michael Tiemann

Received: 9 June 2015 / Accepted: 19 October 2015 / Published: 23 October 2015

\begin{abstract}
We report the results of composite tungsten oxide nanowires-based gas sensors. The morphologic surface, crystallographic structures, and chemical compositions of the obtained nanowires have been investigated using scanning electron microscopy (SEM), X-ray diffraction (XRD), and Raman scattering, respectively. The experimental measurements reveal that each wire consists of crystalline nanoparticles with an average diameter of less than $250 \mathrm{~nm}$. By using the synthesized nanowires, highly sensitive prototypic gas sensors have been designed and fabricated. The dependence of the sensitivity of tungsten oxide nanowires to the methane and hydrogen gases as a function of time has been obtained. Various sensing parameters such as sensitivity, response time, stability, and repeatability were investigated in order to reveal the sensing ability.
\end{abstract}

Keywords: tungsten oxide composite nanowires; gas sensor; sensitivity; response

\section{Introduction}

Oxide semiconductor films exhibit excellent properties for sensor devices [1-5]. There is a strong interest in the development of lightweight gas sensors capable of low ppm range sensitivity and extended 
operation at low-power levels. Recent experimental results demonstrate that the gas-sensing process is strongly related to surface reactions. Different metal oxide-based materials have different reaction (selectivity) activations to the target gases. Consequently, they have a potential for detecting various gases.

When considering the influence factors on gas-sensing properties of metal oxides, it is necessary to reveal their sensing mechanism. The fundamental mechanisms that cause a gas response are still controversial, but are thought to be essential to the trapping of electrons at adsorbed molecules that induces band bending, resulting in a change in conductivity. Moreover, the reaction of species with reducing gases or a competitive adsorption and replacement of the adsorbed species by other molecules decreases and can reverse the band bending, resulting in the variation of conductivity [6,7].

A variety of techniques for the deposition of oxide semiconductors have been developed [8-11]. In general, composite metal oxide sensors exhibited significantly higher sensitivity than conventional sensors constructed solely from one material when tested under identical experimental conditions [12].

Nanostructures have been emphasized in the fabrication of gas-sensitive sensors $[13,14]$. This is in part due to their large surface/volume ratio. For example, Zeng used nano-coral-like porous crystalline $\mathrm{WO}_{3}$ film with a grain size of $9.3 \mathrm{~nm}$ for a gas sensor detecting $\mathrm{NO}_{2}$ at a low operating temperature of $150{ }^{\circ} \mathrm{C}$ [15]. Lee fabricated $\mathrm{WO}_{3}$ a nano-nodule-decorated carbon nanofiber (CNF)-based gas sensor [16]. It was found that the sensitivity of the hybrid CNF gas sensors increased with the decreasing diameter of the CNFs; the minimum detectable level (MDL) was 1 ppm of $\mathrm{NO}_{2}$ gas. Recently, Warmer studied the temperature-dependent sensing properties of metal-oxide semiconductor gas sensors based on $\mathrm{SnO}_{2}$ and $\mathrm{WO}_{3}$, to measure different target gases [17]. However, the requirement of a heater results in a large and complicated structure, causing some existing sensors to become limited either in their range of application or their sensitivity. George Fine and Jin Huang provided an overview of important contributions and recent advances for the use of metal oxide semiconductor sensors for the detection of a variety of gases, respectively $[18,19]$. The nature of the gas response and how it is fundamentally linked to the surface structure was explored. Izadyar theoretically studied cyclic nanostructures of tungsten oxide as a sensing material for gas sensors [20].

Based on these achievements above, the present paper focuses on designing and developing simple, low-cost, room-temperature gas sensors based on tungsten oxide composite nanowires. The nanowires were straightly deposited onto a pair of electrodes. No post-growth processing was needed. We directly used as-synthesized samples to pursue the gas-sensing test. No sample destruction had to be operated. This is important for the commercial usage of gas-sensing device manufacturing. The sensing behaviors of the nanowire arrays to different gases at different temperatures have been examined. Response time and recovery time down to few seconds have obtained at the target gas concentration of $10 \mathrm{ppm}$, whereas at $2 \mathrm{ppm}$, the response time becomes slow up to $1 \mathrm{~min}$. Furthermore, it was found that the fabricated sensor responded to the methane gas by increasing its resistance, whereas it responded to the hydrogen gas by decreasing its resistance.

\section{Experimental Setup}

The nanostructured tungsten oxide materials were synthesized using a simple hot-filament Chemical Vapor Deposition (CVD) technique. The details of the process are described elsewhere in our previous 
publications $[10,21]$. The tungsten filament acted as a precursor for tungsten oxide, and no catalyst or other tungsten-containing compound precursor was used. Both $\mathrm{AlN}$ and $\mathrm{Al}_{2} \mathrm{O}_{3}$ ceramic substrates were used. Prior to the experiments, the substrates were ultrasonically washed in a methanol solution for $5 \mathrm{~min}$, and dried with helium. After placing the substrate, the chamber was pumped down to $2.0 \times 10^{-5}$ Torr, and then fed with the Ar gas (99\%) and oxygen gas (1\%) to 300 mTorr pressure. During deposition, the gas inside the chamber was in a static state. The distance between the hot filament and the substrate remained unchanged. The substrate temperature was controlled by adjusting the electrical current on the hot filament, which is different from our previous experiments where the substrate temperature is controlled by an additional heater under the substrate. Consequently, the present experimental set up was very simple.

\section{Characterizations of the Tungsten Oxide Composite Nanowires}

Traditionally, synthesized tungsten oxide nanowires on a ceramic substrate would be scratched onto the metal fringes to form a simple gas sensor. Preliminary experiments were conducted, but it was found that the electrical contacts between the nanowires and the metal fringe electrode arrays were poor, causing low sensitivity in the obtained sensor. Therefore, in the present paper, the doped tungsten oxide composite nanowires are directly deposited onto a pair of metal electrodes as shown in Figure 1.

The sensor is comprised of a pair of electrodes, nanostructured metal oxide semiconducting wires, a heater, a power supply, and an electrical meter. We fabricated both single-wire- and multi-wire-based gas sensors as shown in Figure 1. No obvious differences were found from the preliminary characterization of two gas sensors. Therefore, the focus of the paper is on the multi-wire-based gas sensors. We used metal platinum as electrodes prepared by using the sputtering deposition technique for the sensor. The thickness of the electrode is up to $3 \mu \mathrm{m}$.

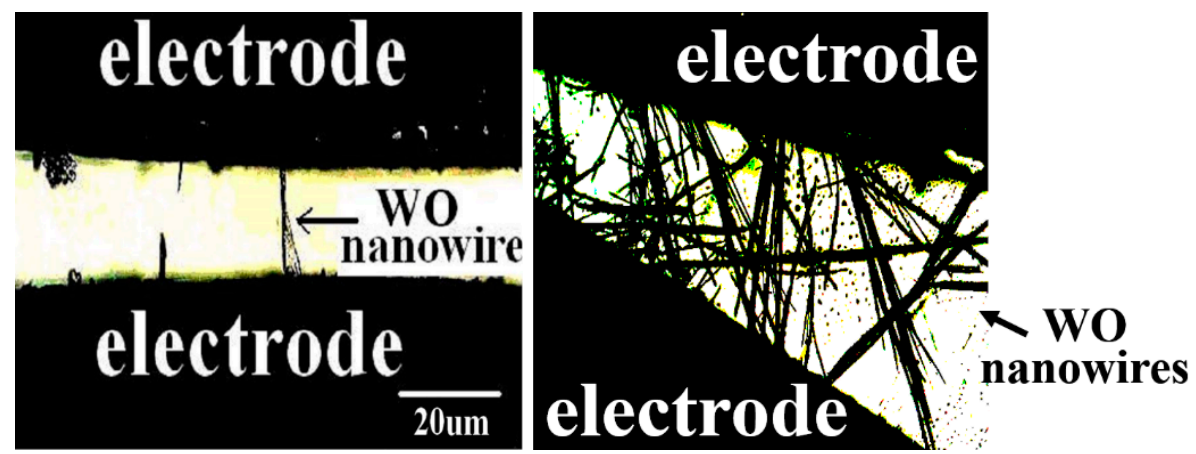

Figure 1. Microscope photograph of the architecture of the prototypical sensor where the nanowires are directly deposited onto a pair of electrodes.

Figure 2 shows typical SEM images of the tungsten oxide nanowires at different magnifications. Each as-grown wire normally consists of a large amount of nanoparticles. The well-shaped edge of each particle is clearly visible as shown in Figure 2a, indicating a crystalline structure. The average size of each particle is $250 \mathrm{~nm}$. The average diameter of the wire is around $500 \mathrm{~nm}$, and the average length of the nanowire is up to $1 \mathrm{~mm}$.

Raman scattering spectrum of the nanowires was obtained at room temperature by using a triple monochomator (ISA J-Y Model T64000, Louisville, KY, USA) with an excitation wavelength of $514 \mathrm{~nm}\left(\mathrm{Ar}^{+}\right.$ion laser). The samples were scanned from $10 \mathrm{~cm}^{-1}$ to $1200 \mathrm{~cm}^{-1}$ as shown in Figure 3 a. 
Several peaks marked with $\mathrm{J}$ and $\mathrm{k}$ in the Raman spectrum were identified. In general, the bands situated at around 700 and $800 \mathrm{~cm}^{-1}$ can be assigned to $\mathrm{W}-\mathrm{O}$ stretching modes, whereas the bands situated at around 130 and $270 \mathrm{~cm}^{-1}$ are associated to $\mathrm{W}-\mathrm{O}$ bending modes of monoclinic $\mathrm{WO}_{3}$ [22]. It was also reported that $\mathrm{J}$ bands at 270 and $330 \mathrm{~cm}^{-1}$ can also be assigned to $\mathrm{W}-\mathrm{O}$ bending modes of monoclinic $\mathrm{WO}_{3}[21,23]$. In our case, $\mathrm{J}_{1}, \mathrm{~J}_{2}$ and $\mathrm{J}_{3}$ bands in Raman spectra of all our tungsten oxide samples always coexist. Therefore, the present three bands have been assigned to $\mathrm{J}$ bands associated with $\mathrm{W}-\mathrm{O}$ bending modes of monoclinic $\mathrm{WO}_{3}$.
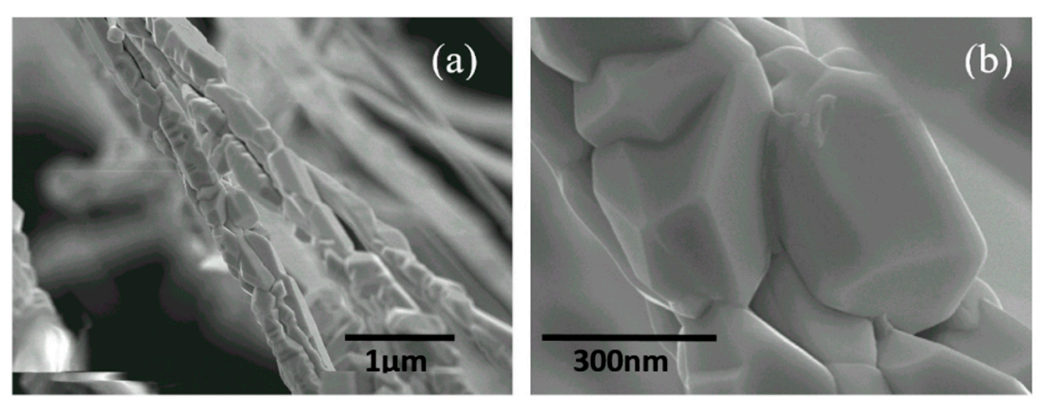

Figure 2. (a) Typical SEM images of tungsten oxide composite nanowires, and (b) magnified SEM images of selected areas.

No obvious shifts of the spectral $\mathrm{k}$ bands of nanowires were observed after the comparison of spectra of the bulk WO material, indicating that the particles inside the nanowires have less stress. However, the variations of content of $\mathrm{WO}_{2}$ and $\mathrm{W}$ in the nanowire composite unavoidably change the crystal symmetry of $\mathrm{WO}_{3}$ that is qualitatively associated with the increasing width of the band. This is confirmed with X-ray Diffraction (XRD) data.
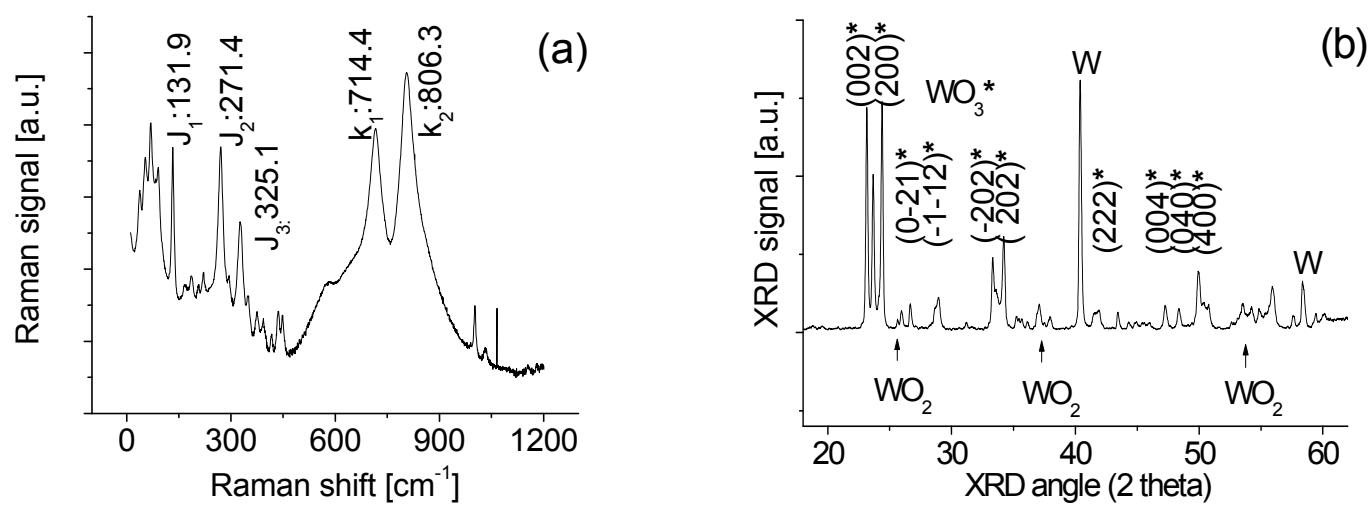

Figure 3. (a) Raman; and (b) XRD of tungsten oxide composite nanowires.

$\mathrm{XRD}$ measurements were conducted to characterize the crystalline structure as shown in Figure $3 \mathrm{~b}$. $\mathrm{WO}_{2}, \mathrm{WO}_{3}$ and $\mathrm{W}$ peaks in XRD spectrum of the nanowires have been identified based on previous literature $[21,23]$. The sample exhibits mixed states including $\mathrm{WO}_{2}, \mathrm{WO}_{3}$ and $\mathrm{W}$. The poly-crystalline $\mathrm{WO}_{3}$ and metal $\mathrm{W}$ dominate the composite of the sample. This is because the intensities of the XRD peaks of $\mathrm{W}$ and $\mathrm{WO}_{3}$ are much stronger than that of the $\mathrm{WO}_{2}$ spectral lines. 


\section{The Results of Fabrication and Characterization of Tungsten Oxide Composite Nanowires-Based Gas Sensors}

The prototypic gas sensor is fabricated based on electrodes, nanowires $\left(R_{S}\right)$, a precise resistor $\left(R_{P}\right)$, a power supply $\left(\mathrm{V}_{\mathrm{p}}\right)$, and an electrical meter $\left(\mathrm{V}_{\mathrm{m}}\right)$ connected in series to form a resistance, current, and voltage (R-I-V) electrical circuit. The variation of the electrical current is caused by incoming external molecules of targeted gas that change the nanowire conductivity. From the measurement of variation of voltage $\left(\mathrm{V}_{\mathrm{m}}\right)$ across the precise resistor $\mathrm{R}_{\mathrm{P}}=8.2 \mathrm{k} \Omega$, the variation of the conductivity of the sensor can be immediately obtained by $R_{s}=\left(V_{p}-V_{m}\right) R_{p} / V_{m}$. Necessary calibrations of the sensor conducted at the characterization chamber are shown in Figure 4. It includes a plasma beam source for the treatment of the surface of the sensor device, the mass flow meter (Model FM-360, Tylan Corporation), a precise pressure gauge, a mass spectrometer, etc., for different experimental purposes.

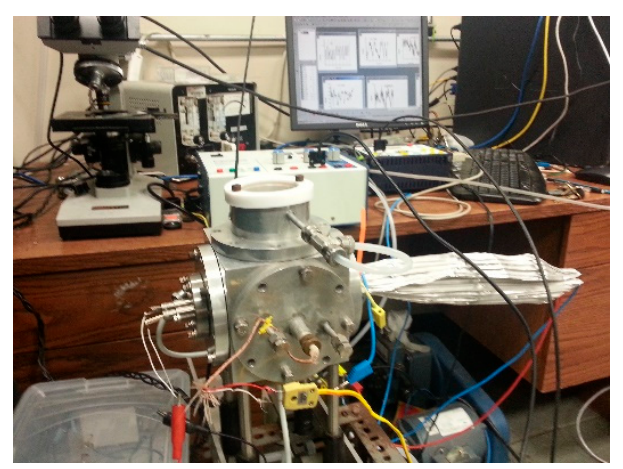

Figure 4. Photograph of experimental set up for characterization of newly fabricated gas sensors.

An additional tank was also used to mix the target gas (methane or hydrogen) with nitrogen gas, and two Omega mass flow controllers (one for the target gas and other one for the nitrogen gas) were installed to control target gas concentrations. Because of local high-humidity weather and because we did not desire such a humidity effect on the present sensor, we did not mix the target gas with air. A detailed discussion of the humidity effect can be found in our previous papers [24,25].

The characterizations of the properties of the fabricated sensors include the sensitivity/resistance $\left(\mathrm{R}_{\mathrm{s}}\right)$,

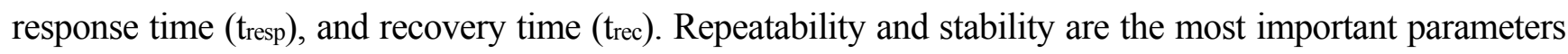
for a gas sensor. The gas response $(\mathrm{S})$ is defined by directly using the impedance of the sensing material in the fabricated sensor. We did not use a traditional definition of $\left(\mathrm{R}_{\text {gas }}-\mathrm{R}_{\text {nitrogen }}\right) / \mathrm{R}_{\text {nitrogen }}$ for the response because it could have yielded a negative value for the response when $\mathrm{R}_{\text {gas }}$ is less than $\mathrm{R}_{\text {nitrogen. }}$.

Figure 5 shows the typical response at room temperature when the nanowire array-based gas sensor is cycled between the "switch-on" and "switch-off" of exposure to the methane gas. The concentration of the gas molecule is $2 \mathrm{ppm}$. The sensor has good features in repeatability and stability. It responded to the methane gas by increasing its resistance. The changes in conductivity/resistance can be attributed to the adsorption of methane molecules. Since the shift of the spectral line is observed, it is expected that the adsorption type of methane molecules is chemisorption.

The response and recovery times of the sensors are about $1.5 \mathrm{~min}$ and $1 \mathrm{~min}$, respectively. The definition is based on the time duration to reach $90 \%$ of the full response of the sensor. It is found that the response-recovery time depends on the nature of the samples, gas concentration, and operating temperature 
of sensors. In the case where the targeted gas is of low concentration, the time response and recovery are delayed. This can be explained by the fact that there are not enough molecules participating in the reaction with the nanoparticles, and therefore more time is needed for reaching the balance of the reaction between the particle and the targeted molecular gas. In contrast, for the high concentration case, far less time is needed to reach the equilibrium of the reaction. Consequently, short response time can be obtained.

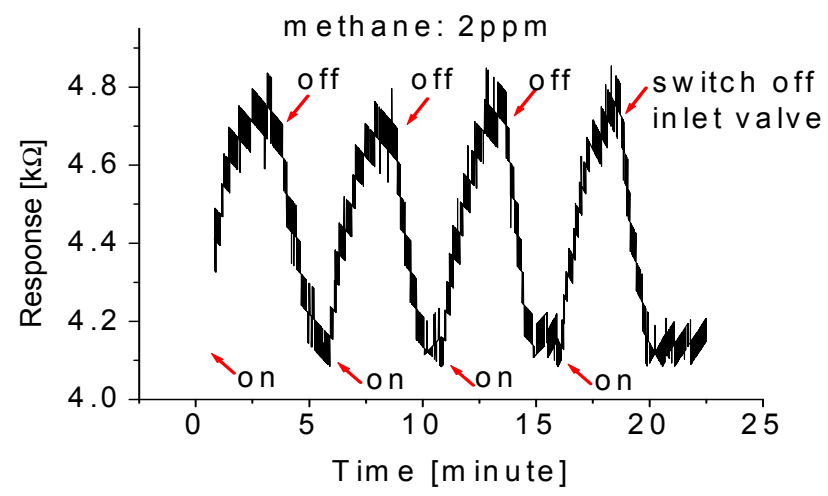

Figure 5. Typical response at room temperature when the nanowire array-based gas sensor is cycled between the "switch-on" and "switch-off" of exposure to the methane gas with a gas concentration of $2 \mathrm{ppm}$.

Figure $6 \mathrm{a}$ depicts the response of the fabricated gas sensor tested at the methane concentrations of $10 \mathrm{ppm}, 6 \mathrm{ppm}$, and $2 \mathrm{ppm}$, respectively. The measurements were performed at room temperature. To test the sensor, it is first exposed to $10 \mathrm{ppm}$ methane for $180 \mathrm{~s}$ and then the valve was switched off for $120 \mathrm{~s}$. This was then repeated every $5 \mathrm{~min}$ with the concentration decreased in steps of $4 \mathrm{ppm}$ for each cycle. An obvious change of electrical resistance of the nanowires was found upon exposure to the methane gas. The variation of resistance was around $1.9 \mathrm{k} \Omega$ at $10 \mathrm{ppm}$ of the target gas, $1.3 \mathrm{k} \Omega$ at $6 \mathrm{ppm}$, and $0.6 \mathrm{k} \Omega$ at $2 \mathrm{ppm}$. This possibly indicates that the output change (or resistance change) of the prototypical sensor has an almost linear relationship with the methane concentration within a small area ranging from $2 \mathrm{ppm}$ to $10 \mathrm{ppm}$. However, we are not able to conclude the trend of the resistance change as a function of the concentration of methane from the limited data.
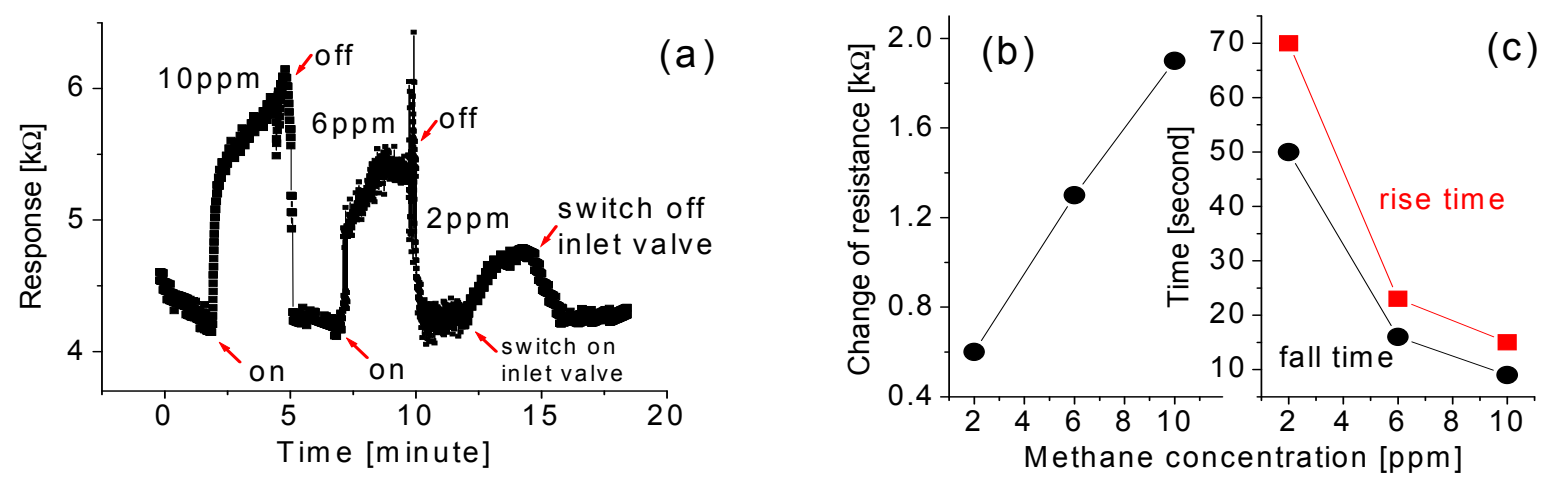

Figure 6. (a) The response of the fabricated gas sensor tested at the methane concentrations of 10 ppm, 6 ppm, and 2 ppm, respectively, at room temperature; (b) output change (or resistance change); and (c) the rise time and fall time as a function of the methane concentrations. 
It is also found that the recovery time is much shorter in the case of high concentration than that of low concentration. For example, in the $10 \mathrm{ppm}$ case, the recovery time is only a few seconds as shown in Figure 6c, which is much shorter than the recovery time of the conventional sensors which are usually about $100 \mathrm{~s}$ and more than $500 \mathrm{~s}$, respectively. It is relatively difficult to precisely identify the rise time because the profile can be divided into several areas. According to Figure 6a, the response time (the definition is based on the time duration to reach $70 \%$ of the full response of the sensor) of the gas sensors is short, as depicted in Figure 6c. However, if the definition is based on the time duration to reach $90 \%$ of the full response of the sensor, the response time is longer.

It is noted that both response and recovery times are delayed with the decrease in the concentration of the target gases. Desorption would be easier in an atmosphere with a low concentration of gas, and the recovery time should be shorter when the response time is prolonged. Furthermore, a faster recovery time is possibly related to the use of pumping, which could significantly accelerate the desorption process.

The operating temperature greatly influences the properties of sensing nanowire materials. Therefore, before characterizing the temperature effect on the sensing behavior such as sensitivity and response time, the relationship between the resistance of the nanowires and the temperature was determined. Figure 7 shows the dependence of the resistance of the tungsten oxide nanowires as a function of temperature. Clearly, the resistance of the nanowires reduces from $4.1 \mathrm{k} \Omega$ to $2.8 \mathrm{k} \Omega$ with an increase of the temperature from $20^{\circ} \mathrm{C}$ to $100^{\circ} \mathrm{C}$. The characterizations of electrical properties were also conducted at $20^{\circ} \mathrm{C}, 100{ }^{\circ} \mathrm{C}$, and $200{ }^{\circ} \mathrm{C}$, as shown in Figure $7 \mathrm{~b}$. Two conclusions can be made based on the data in Figure 7b: (1) at a fixed temperature, the electrical current and voltage always have a linear relationship, indicating the ohm contact between the electrodes and the nanowires, and that there is no more charge accumulation or polarization effect in the circuit; and (2) the electric properties of the nanoparticle-based nanowires are sensitive to temperature. The reason of this is that zero-dimension structures like nanoparticles have much strong mutual link condition. Thus, the intrinsic structure of nanoparticles will undergo a great change with temperature.
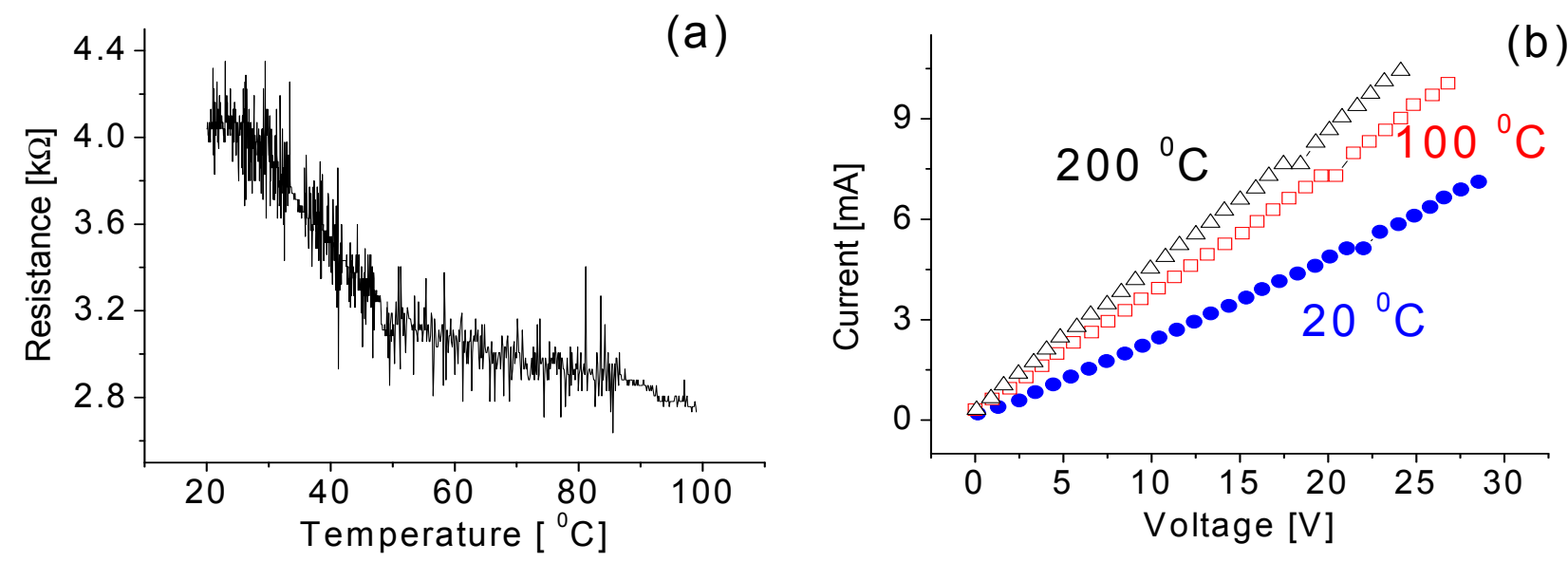

Figure 7. (a) Variation of resistance of the nanowires following the increase of temperature; and (b) the temperature effect on current-voltage curves.

Temperature effects on the responses of the sensor are depicted in Figure 8. When the sensor was exposed to the $2 \mathrm{ppm}$ methane gas at an operating temperature of $50{ }^{\circ} \mathrm{C}$, the output of the sensor increased 
and then reached a relatively stable value. When the inlet valve for the methane gas was switched off, the resistance abruptly decreased and then gradually reached a relatively stable value. Good reproducibility and short recovery time down to a few seconds have been obtained. However, no obvious improvement for the response time was observed. A similar phenomenon was also observed for the case at the operating temperature of $75^{\circ} \mathrm{C}$ as shown in Figure $8 \mathrm{~b}$. A likely reason explaining why there is no outstanding rise time is the time needed for the gas to diffuse until reaching a balanced state inside the chamber.
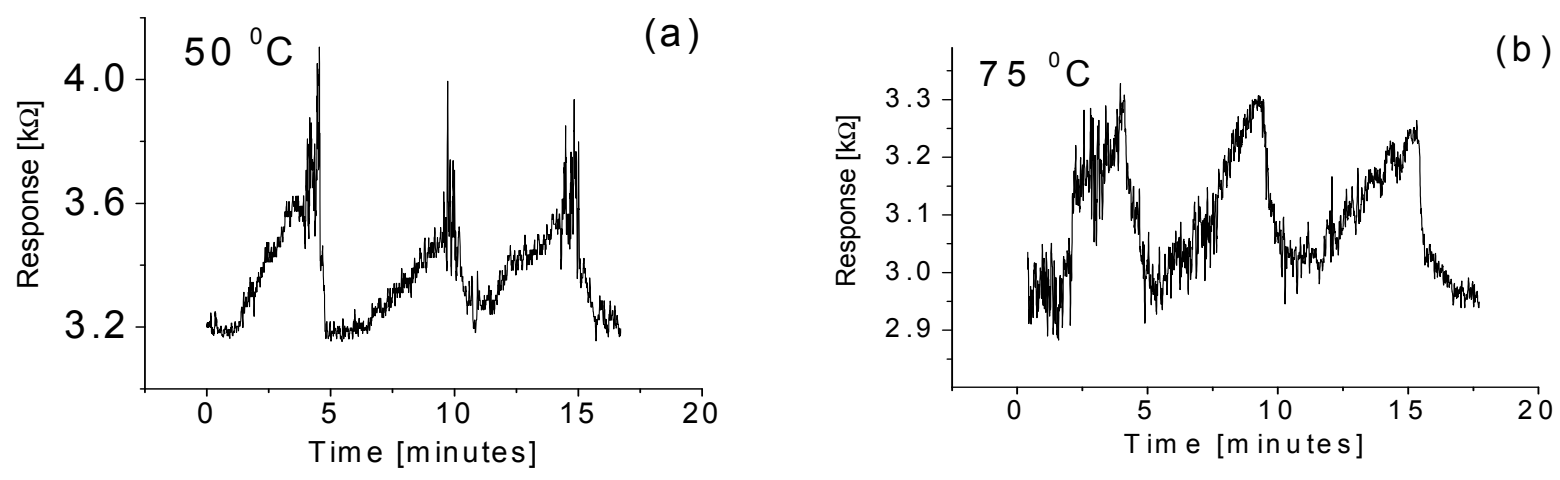

Figure 8. Responses of the sensor to the methane gas with a 2 ppm concentration at operating temperatures of (a) $50{ }^{\circ} \mathrm{C}$; and (b) $75^{\circ} \mathrm{C}$.

It is important to point out that good baseline stability is always visible at any operating temperature, but the resistance/output of the response of the sensor is slightly affected after several exposures to the methane gas at high temperatures, as shown in Figure 8. A slight drop in the output (around approximately $1 \%$ ) is observed when the sensor is exposed to the targeted gas every cycle. Such a drop is possibly due to chemisorption of molecules, which is responsible for changes in the electrical resistance of metal oxide at relative high temperatures due to the incomplete desorption of methane molecules on the surface of the nanowire sensor.

As a comparison, the fabricated sensor is also employed for detecting hydrogen gas. It was found that the response of the fabricated sensor to the hydrogen gas was completely different from that to the methane gas. Figure 9 shows responses at room temperature when the sensor is cycled between the "switch-on" and "switch-off" of exposure to the hydrogen gas with the gas concentration of 20 ppm and $2 \mathrm{ppm}$, respectively. Important stability or repeatability features obtained from the cycled test are clearly visible. The tungsten oxide composite-based gas sensor responded to $20 \mathrm{ppm}$ hydrogen gas by decreasing its resistance, and then quickly reached a relatively stable value. The response time of around $5 \mathrm{~s}$ and the recovery time of around $10 \mathrm{~s}$ have been obtained.

When the hydrogen concentration decreases down to $2 \mathrm{ppm}$, the output of the signal strength drops almost 90\% as shown in Figure 9b. Furthermore, both the rise time and fall time extend to around $1.5 \mathrm{~min}$. This phenomenon is similar to that of the sensor exposure to the methane gas. The possible reason is that not enough molecules are adsorbed onto the surface of the nanowires for the reaction.

It is generally known that the gas-sensing mechanism is based on the interaction between the negatively charged oxygen adsorbed on the tungsten oxide surface and the targeted gas [26]. When the oxide semiconductor-based sensor is exposed to $\mathrm{H}_{2}$, hydrogen molecules react with these adsorbed atoms 
to increase the conductivity of the WO-based sensor by releasing electrons as shown in Figure 9. However, such an interpretation cannot be used to explain the response of the sensor to the methane gas as shown in Figures 5 and 8.
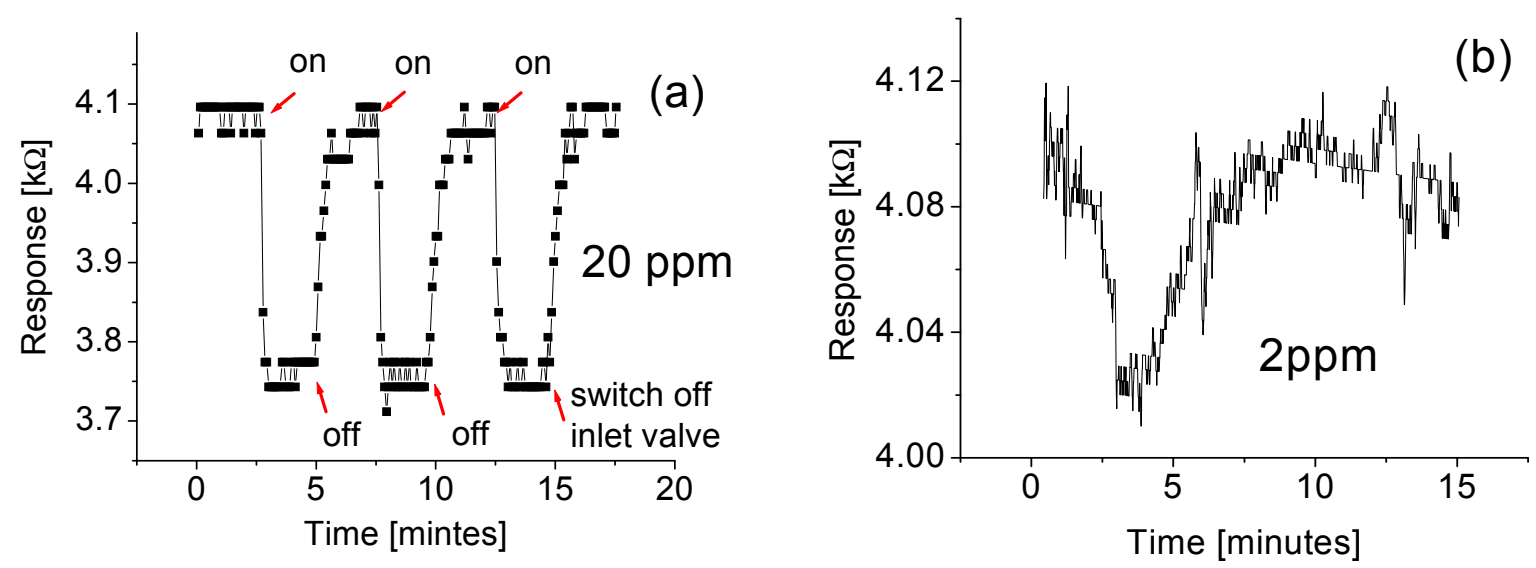

Figure 9. Responses of the sensor to the hydrogen gas at room temperature with the concentration of (a) 20 ppm; and (b) 2 ppm.

It is noticed from the XRD spectrum of the synthesized nanowires in Figure $3 b$ that there is a large amount of metal W component mixed with the WO material. If the sensor is exposed to the methane gas, these gas molecules would not only react with the negatively charged oxygen adsorbed on the tungsten oxide surface but also with the metal $\mathrm{W}$ component. Once the targeted gas molecules and the metal $\mathrm{W}$ dominated the reaction, it would result in reducing conductivity as shown in Figure 5. This change in the sensitivity could be attributed to a synergistic or competitive effect. Here it should be mentioned that analyses of the data obtained from additional experiments with higher concentrations of $\mathrm{W}$ indicated that a higher output/response of the fabricated sensor to methane gas has been obtained. In contrast, it does not significantly affect the sensor's response to hydrogen gas. This evidence supports our explanation of the gas-sensing mechanism that when the targeted gas molecules and the metal tungsten dominated the reaction, it would result in the decrease of conductivity or an increase of its resistance.

\section{Conclusions}

We conclude that each as-grown wire mainly consists of a large amount of nanoparticles. The sample exhibits mixed states including $\mathrm{WO}_{2}, \mathrm{WO}_{3}$, and $\mathrm{W}$. The metal $\mathrm{W}$ and poly-crystalline $\mathrm{WO}_{3}$ dominate the composition of the sample. Experimental data indicate that composite nanowires directly deposited onto a pair of electrodes can improve the performance of gas sensors such as good repeatability and stability. The response and recovery times of the fabricated sensors at 2 ppm concentration are about 1.5 min and 1 min, respectively. In contrast, it is only few $\mathrm{s}$ at the concentration of $10 \mathrm{ppm}$.

The operating temperature has a great influence on the properties of the sensor. A quick recovery time down to a few seconds has been observed, but there is no obvious improvement in response time. The reason why there is no outstanding rise time can be attributed to the time needed by the gas to diffuse itself until reaching a balanced state inside the chamber. 
Differing from the response to the methane gas, the fabricated sensor responded to hydrogen gas by decreasing its resistance. Response and recovery time to the hydrogen gas are less than $5 \mathrm{~s}$ operated at $20 \mathrm{ppm}$, and $1.5 \mathrm{~min}$ at $2 \mathrm{ppm}$.

The general interpretation for the gas-sensing mechanism is based on the interaction between the negatively charged oxygen adsorbed on the surface of the composite and the targeted gas. Since there is a large amount of metal $\mathrm{W}$ component mixed with the WO material in the present case, if the sensor is exposed to the targeted gas, these gas molecules will not only react with the negatively charged oxygen adsorbed on the tungsten oxide surface, but they will also react with the metal $\mathrm{W}$ component. The change in the sensitivity is attributed to a synergistic or competitive effect.

\section{Acknowledgments}

Siyuan Feng-Chen acknowledges the support from Army research office/DoD HSAP program. Ali Aldalbahi acknowledges the financial support from Deanship of Scientific Research at King Saud University (RG-1436-005). Peter Xianping Feng acknowledges the support of Visiting Professor Program and DoD grant (62826-RT-REP).

\section{Author Contributions}

Siyuan Feng-Chen presented parts of the work at the San Juan Metropolitan Science Fair 2015. She contributed to the conducting experiments, data analysis, and writing of the manuscript. Ali Aldalbahi provided a contribution on sample characterization, data interpretation, and manuscript preparation. Peter Xianping contributed to the data analysis, data interpretation, and jointly writing the manuscript. All authors contributed to design of the experiments, discussion, and analyses of the results in the manuscript.

\section{Conflicts of Interest}

The authors declare no conflict of interest.

\section{References}

1. Akhtar, K.; Haq, I.U.; Malook, K. Gas sensing properties of semiconducting copper oxide nanospheroids. Powder Technol. 2015, 283, 505-511.

2. Trakhtenberg, L.I.; Gerasimov, G.N.; Gromov, V.F.; Belysheva, T.V.; Ilegbusi, O.J. Gas semiconducting sensors based on metal oxide nanocomposites. J. Mater. Sci. Res. 2012, 1, 56-68.

3. Chu, J.; Peng, X.; Sajjad, M.; Yang, B.; Feng, P.X. Nanostructures and sensing properties of ZnO prepared using normal and oblique angle deposition techniques. Thin Solid Films 2012, 520, 3493-3498.

4. Moos, R.; Sahner, K.; Fleischer, M.; Guth, U.; Barsan, N.; Weimar, U. Solid state gas sensor research in Germany-A status report. Sensors 2009, 9, 4323-4365.

5. Yamazoe, N.; Shimanoe, K. New perspectives of gas sensor technology. Sens. Actuators B Phys. 2009, 138, 100-107. 
6. Henrich, V.E.; Cox, P.A. The Surface Science of Metal Oxides; Cambridge University Press: Cambridge, UK, 1994.

7. Wang, C.; Yin, L.; Zhang, L.; Xiang, D.; Gao, R. Metal oxide gas sensors: Sensitivity and influencing factors. Sensors 2010, 10, 2088-2106.

8. Chu, J.; Peng, X.Y.; Dasari, K.; Palai, R.; Feng, P. The shift of optical band gap in W-doped ZnO with oxygen pressure and doping level. Mater. Res. Bull. 2014, 54, 73-77.

9. Peng, X.; Boqian, Y.; Chu, J.; Feng, P. Effects of nitrogen pressure during pulsed laser deposition on morphology and optical properties of N-doped ZnO nanostructures. Surf. Sci. 2013, 609, 48-51.

10. Peng, X.; Chu, J.; Feng, P. Synthesis of WOx-ZnO double-layer by two step process. Mater. Lett. 2012, $73,65-68$.

11. Moezzi, A.; McDonagh, A.M.; Cortie, M.B. Zinc oxide particles: Synthesis, properties and applications. Chem. Eng. J. 2012, 185-186, 1-22.

12. Göpel, W.; Schierbaum, K.D. $\mathrm{SnO}_{2}$ sensors - Current status and future prospects. Sens. Actuators $B$ Chem. 1995, 26, 1-2.

13. Cho, P.S.; Kim, K.W.; Lee, J.H. $\mathrm{NO}_{2}$ sensing characteristics of $\mathrm{ZnO}$ nanorods prepared by hydrothermal method. J. Electroceram. 2006, 17, 975-978.

14. Wang, H.T. Kang, B.S.; Ren, F. Hydrogen-Selective sensing at room temperature with $\mathrm{ZnO}$ nanorods. Appl. Phys. Lett. 2005, 86, 243503.

15. Zeng, J.; Hu, M.; Wang, W.; Chen, H.; Qin, Y. $\mathrm{NO}_{2}$-Sensing properties of porous $\mathrm{WO}_{3}$ gas Sensor based on anodized sputtered tungsten thin film. Sens. Actuators B Chem. 2012, 161, 447-452.

16. Lee, J.S.; Kwon, O.S.; Shin, D.H.; Jang, J. $\mathrm{WO}_{3}$ Nanonodule-decorated hybrid carbon nanofibers for $\mathrm{NO}_{2}$ gas sensor application. J. Mater. Chem. A 2013, 1, 9099-9106.

17. Warmer, J.; Wagner, P.; Schöning, M.J.; Kaul, P. Detection of triacetone triperoxide using temperature cycled metal-oxide semiconductor gas sensors. Phys. Status Solidi A 2015, 212, 1289-1298.

18. Fine, G.F.; Cavanagh, L.M.; Afonja A.; Russell, B. Metal oxide semi-conductor gas sensors in environmental monitoring. Sensors 2010, 10, 5469-5502.

19. Huang, J.; Wan, Q. Review gas sensors based on semiconducting metal oxide one-dimensional nanostructures. Sensors 2009, 9, 9903-9924.

20. Izadyar, M.; Jamsaz, A. Cyclic nanostructures of tungsten oxide as gas sensor: A theoretical study. Int. J. Anal. Chem. 2014, 2014, doi:10.1155/2014/240197.

21. Wang, X.P.; Yang, B.Q.; Zhang, H.X.; Feng, P.X. Tungsten oxide nanorods array and nanobundle prepared by using chemical vapor deposition technique. Nanoscale Res. Lett. 2007, 2, 405-409.

22. Kumar, V.B.; Mohanta, D. Formation of nanoscale tungsten oxide structures and colouration characteristics. Bull. Mater. Sci. 2011, 34, 435-442.

23. Xiao, Z.D.; Zhang, L.D.; Tian, X.K.; Fang, X.S. Fabrication and structural characterization of porous tungsten oxide nanowires. Nanotechnology 2005, 16, 2647.

24. Chu, J.; Peng, X.; Feng, P.; Sheng, Y.; Zhang, J. Study of humidity sensors based on nanostructured carbon films produced by physical vapor deposition. Sens. Actuators B Chem. 2013, 178, 508-513. 
25. Peng, X.; Yang, B.; Chu, J.; Feng, P. Mn-doped zinc oxide nanopowders for humidity sensors. Sens. Actuators B Chem. 2012, 174, 258-262.

26. Chu, J.; Peng, X.; Wang, Z.; Feng, P. Sensing performances of $\mathrm{ZnO}$ nanostructures grown under different oxygen pressures to hydrogen. Mater. Res. Bull. 2012, 47, 4420-4426.

(C) 2015 by the authors; licensee MDPI, Basel, Switzerland. This article is an open access article distributed under the terms and conditions of the Creative Commons Attribution license (http://creativecommons.org/licenses/by/4.0/). 